\title{
Geographic variations in idiopathic epiretinal membranes in China
}

\author{
Mengqiao $\mathrm{Xu}^{1}$, Minwen Zhou ${ }^{1}$, Xiaodong Sun ${ }^{1}$, Xiang Shi ${ }^{2}$ \\ ${ }^{1}$ Department of Ophthalmology, Shanghai General Hospital, National Clinical Research Center for Eye Diseases, Shanghai Key Laboratory of \\ Fundus Disease, Shanghai Engineering Center for Visual Science and Photomedicine, Shanghai Engineering Center for Precise Diagnosis and \\ Treatment of Eye Diseases, Shanghai Jiao Tong University School of Medicine, Shanghai, China; ${ }^{2}$ Department of Ophthalmology, Affiliated Hospital \\ of Nantong University, Nantong, China \\ Contributions: (I) Conception and design: X Shi, X Sun; (II) Administrative support: M Zhou; (III) Provision of study materials or patients: M Xu; (IV) \\ Collection and assembly of data: M Xu, X Shi; (V) Data analysis and interpretation: M Xu; (VI) Manuscript writing: All authors; (VII) Final approval \\ of manuscript: All authors. \\ Correspondence to: Xiang Shi. Department of Ophthalmology, Affiliated Hospital of Nantong University, Nantong, China. Email: drshi2012@foxmail.com.
}

\begin{abstract}
Background: Idiopathic epiretinal membranes (ERMs) often cause metamorphopsia and the progressive loss of central visual function, which seriously affect quality of life. We aimed to map the distribution pattern of idiopathic ERMs in China and to examine the factors affecting the surgical choices of multicenter surgeons. Methods: A national ophthalmologist-oriented questionnaire was administered, applied with a multistage probability sampling method. Data of essential characteristics, including age, professional title, residence, and perioperative and postoperative care, were gathered. All the data are expressed as odds ratios (ORs) and 95\% confidence intervals (CIs). The histogram and choropleth map were generated by Excel 2016.

Results: In total, 1,137 (85.2\%) valid responses were returned with maximized response and completion rates. The study showed that monthly admission numbers, and preoperative and postoperative care varied significantly across different regions in China. Generally, the monthly patient admission numbers were lower in the Western region than the Eastern region. However, patients in the Eastern region had longer preoperative waiting periods and shorter hospital stays.

Conclusions: The epidemiology of idiopathic ERMs varied significantly across different regions in China. The distribution pattern of ERM in China and the overview of the factors affecting the surgery approaches of multicenter surgeons were shown. The findings of this study will contribute to the formulation of medical policies, and provide insights into the healthcare environments across China.
\end{abstract}

Keywords: Idiopathic epiretinal membrane; questionnaire; epidemiologic survey; medical policy

Submitted Apr 08, 2021. Accepted for publication Jun 09, 2021.

doi: $10.21037 / \mathrm{atm}-21-2722$

View this article at: http://dx.doi.org/10.21037/atm-21-2722

\section{Introduction}

An idiopathic epiretinal membrane (ERM) is characterized by a semi-translucent epimacular membranous tissue, and is caused by cellular proliferation at the vitreoretinal interface (1). The precise pathology of an ERM includes the proliferation of different cell types, such as glial cells, hyalocytes, and fibroblasts, followed by abnormal posterior vitreous detachment, and vitreoschisis (2,3). After membrane formation, the contraction and shrinkage of the proliferative membrane exert anteroposterior and tangential tractional forces on the retinal tissue and vasculature $(4,5)$. An ERM may result in macular distortion, thus inducing metamorphopsia and the progressive loss of central visual function, which seriously affect quality of life.

Several population-based epidemiology studies have sought to estimate the prevalence of ERM. The incidence of ERM has been reported to vary vastly from $1.0 \%$ to $28.9 \%$ among different ethnic groups. Most studies have focused on Caucasian people (6). However, national epidemiological data for ERMs among the Chinese population are limited. A number of studies have reported regional population- 
based ERM prevalence data in economically developed areas, such as Shanghai and Beijing; however, the incidence rates reported have ranged from $1.0 \%$ to $8.4 \%$ (7).

Additionally, ERMs are unlikely to be resolved without surgical intervention. Vitrectomy and the removal of ERMs accompanied by internal limiting membrane (ILM) peeling is one of the most effective treatments for ERMs, and can remarkably improve the subjective perception of visual function and metamorphopsia, even in the absence of significant visual acuity improvement (8). Its surgery efficacy for ERMs has been validated (9). However, those studies have mainly focused on prognostic factors after surgery. To date, only limited studies have been conducted on geographic variations in ERM surgery (e.g., variations in surgical methods and surgery timing) among the Chinese national population. We aimed to map the distribution pattern of ERM in China and to provide an overview of the factors affecting the surgery approaches of multicenter surgeons, thus providing a pilot national dataset. Specifically, we sought to conduct a national investigation of ERM care (NIEC) based on a questionnaire.

We present the following article in accordance with the SURGE reporting checklist (available at http://dx.doi. org/10.21037/atm-21-2722).

\section{Methods}

\section{Participants and methods}

The conduct the NIEC, a questionnaire was administered by a Shanghai general hospital with one of the biggest ophthalmic centers in China. The questionnaire respondents comprised ophthalmologists rather than patients, who are more commonly used as respondents.

Briefly, a multistage probability sampling method was applied based on previous studies. This study encompassed 30 provincial capital cities in China, including 3 tertiary hospitals in each city. We selected over 10 eligible ophthalmologists at each selected hospital to complete the questionnaire. The study was conducted according to the Declaration of Helsinki (as revised in 2013) and was approved by the Institutional Ethics Board of Shanghai General Hospital (No. [2020]92) and individual consent for this cross-sectional survey was waived.

\section{Questionnaires}

Our research team designed a standardized ophthalmologist- oriented questionnaire to elicit information about essential characteristics, such as age, professional title and residence, and perioperative and postoperative care. The questionnaire included questions relating to monthly admissions of ERM (1-10, 11-20, 21-30, 31-40, or >40), preoperative waiting times $(1-3,4-7,8-14,15-30$, or $>30 \mathrm{~d})$, hospitalization periods $(1-3,4-5,6-7$, or $>7 \mathrm{~d})$, and the choice of a conservative treatment or a vitrectomy with ERM removal and ILM peeling. A web-based questionnaire engine made it easier for respondents to complete the questionnaire.

30 provincial capital cities were categorized into 6 regions (i.e., Eastern, Northern, South-Central, South-Western, North-Eastern, and North-Western). The Eastern region comprised Shanghai, Jinan, Nanjing, Hangzhou, Fuzhou, Hefei, and Nanchang; the Northern region comprised Beijing, Tianjin, Shijiazhuang, Taiyuan, and Huhehaote; the South-Central comprised Zhengzhou, Wuhan, Changsha, Guangzhou, Nanning, and Haikou; the South-Western comprised Chongqing, Chengdu, Guiyang, and Kunming; the North-Eastern comprised Shenyang, Changchun, and Haerbing; and the North-Western comprised Xian, Lanzhou, Xining, Yinchuan, and Wulumuqi (10). The various regions had different socioeconomic statuses (SESs), different climates, and terrains, and a different number of patients, and thus represented the primary conditions all over China. The analyses of ERMs were based on these regions to identify particular factors.

The web-based questionnaire was distributed via a specified website, email, or mobile device to the respondent ophthalmologists simultaneously to maximize the response and completion rates. The returned questionnaires were collected and reviewed by 1 reviewer within 30 days.

\section{Statistical analysis}

All the data are expressed as odds ratios (ORs) and 95\% confidence intervals (CIs). The histogram and choropleth map were generated by Excel 2016. SPSS (SPSS for Windows, version 22.0) software was used for the statistical analysis.

\section{Results}

\section{Participants}

Among the 1,335 email- and mobile-based NIEC questionnaire issued, $1,137(85.2 \%)$ valid responses were returned, including 1,025 (90.1\%) mobile- and 112 
Table 1 The common characteristics of the NIEC questionnaire respondents

\begin{tabular}{|c|c|c|c|c|c|c|c|}
\hline Characteristics & Eastern & Northern & North-Eastern & North-Western & South-Central & South-Western & Total \\
\hline \multicolumn{8}{|l|}{ Age (years), n (\%) } \\
\hline$\leq 30$ & $16(5.4)$ & $24(10.3)$ & $10(9.9)$ & $12(7.8)$ & $25(10.8)$ & $21(17.4)$ & $108(9.5)$ \\
\hline $31-40$ & $211(71.5)$ & $170(72.6)$ & $71(70.3)$ & $97(63.0)$ & $166(71.6)$ & $71(58.7)$ & $786(69.1)$ \\
\hline $51-60$ & $5(1.7)$ & $2(0.9)$ & $3(3.0)$ & $7(4.5)$ & $8(3.4)$ & $1(0.8)$ & $26(2.3)$ \\
\hline$>60$ & 0 & 0 & 0 & 0 & 0 & 0 & $0(0.0)$ \\
\hline \multicolumn{8}{|l|}{ Professional title, n (\%) } \\
\hline Intern & $3(1.0)$ & $6(2.6)$ & $5(5.0)$ & $8(5.2)$ & $4(1.7)$ & $5(4.1)$ & $31(2.7)$ \\
\hline Associate chief physician & $67(22.7)$ & $43(18.4)$ & $30(29.7)$ & $54(35.1)$ & $42(18.1)$ & $36(29.8)$ & $272(23.9)$ \\
\hline Chief physician & $8(2.7)$ & $5(2.1)$ & $6(5.9)$ & $12(7.8)$ & $9(3.9)$ & $4(3.3)$ & $44(3.9)$ \\
\hline
\end{tabular}

NIEC, national investigation of ERM care.

(9.9\%) email-based completed questionnaires. The other questionnaires were ultimately excluded due to email failures, incomplete responses, missing items, or specific errors. The common characteristics of the respondents who completed the NIEC questionnaire are set out in Table 1.

\section{Montbly admissions of ERM patients}

Based on the presented statistical results, the proportion of the monthly admissions of ERM patients in different regions were similar. The Western region accounted for a relatively small proportion of the monthly admissions due to the SES of the region.

To conduct the quantitative comparison among different regions, monthly admissions of over 20 patients were chosen to compare proportions. The South-Western and North-Western regions had the smallest proportions of admissions [13.3\% (16 of 121) and 15.6\% (24 of 154)], followed by the South-Central (36.2\%; 84 of 232), NorthEastern (37.6\%; 38 of 101), Northern (49.2\%; 115 of 234), and Eastern regions $(60.7 \%$; 179 of 295) (see Table 2, and Figures 1 and $2 A$ ).

\section{Preoperative and postoperative care}

The determining factors chosen for the continuative analysis were prognosis and comorbidity after treatment, and preoperative and postoperative care. The preoperative waiting time and the length of hospital stay varied significantly among different regions (Figure $2 B$ ). The Eastern and Northern regions accounted for larger proportions of long waiting times of over 30 days [45.8\% (135 of 295$)$ and $44.9 \%$ (105 of 234), respectively], [note: North-Western region (3.3\% (5 of 154); OR: 25.14 and 24.26; 95\% CI: $10.02-63.10$ and 9.59-61.33; $\mathrm{P}<0.001]$. Additionally, the proportions of hospitalization periods less than 3 days were also compared. The North-Western region accounted for the highest proportion $(73.4 \% ; 113$ of 154), while the Eastern region had the lowest proportion [2.0\% (6 of 295); OR: 132.75; 95\% CI: 54.85-321.29; $\mathrm{P}<0.001$ ] (see Table 2 and Figure 2C,D).

\section{The choice of conservative treatment versus ERM surgery}

Patients with mild symptoms and comorbidities may benefit from conservative treatments; however, other patients might choose conservative treatments for socioeconomic reasons. The peeling of the ILM is applied as an accessory procedure to improve the curative effect. However, some doctors do not use ILM peeling because its functional benefits are debated.

Thus, it is crucial to establish the appropriate standards 
Table 2 Common variables in the treatment of ERM according to the NIEC questionnaire

\begin{tabular}{|c|c|c|c|c|c|c|}
\hline Characteristics & Eastern & Northern & North-Eastern & North-Western & South-Central & South-Western \\
\hline $1-10$ & $14(4.7)$ & $16(6.8)$ & $38(37.6)$ & $98(63.6)$ & $27(11.6)$ & $74(61.1)$ \\
\hline $11-20$ & $102(34.6)$ & $103(44.0)$ & $25(24.8)$ & $32(20.8)$ & $121(52.2)$ & $31(25.6)$ \\
\hline $21-30$ & $140(47.5)$ & $84(35.9)$ & $26(25.7)$ & $21(13.6)$ & $72(31.0)$ & $10(8.3)$ \\
\hline$>40$ & $11(3.7)$ & $10(4.3)$ & $4(4.0)$ & 0 & $2(0.9)$ & 0 \\
\hline \multicolumn{7}{|l|}{ Preoperative waiting time (h), n (\%) } \\
\hline $1-3$ & $6(2.0)$ & $8(3.4)$ & $12(11.9)$ & $113(73.4)$ & $23(9.9)$ & $79(65.3)$ \\
\hline $4-7$ & $12(4.0)$ & $8(3.4)$ & $25(24.7)$ & $21(13.6)$ & $62(26.7)$ & $13(10.7)$ \\
\hline$>30$ & $135(45.8)$ & $105(44.9)$ & $12(11.9)$ & $5(3.3)$ & $52(22.4)$ & $10(8.3)$ \\
\hline \multicolumn{7}{|l|}{ Length of hospitalization (d), n (\%) } \\
\hline $1-3$ & $163(55.2)$ & $104(44.4)$ & $22(21.8)$ & $10(6.5)$ & $26(11.2)$ & $12(9.9)$ \\
\hline $4-5$ & $102(34.6)$ & $107(45.7)$ & $48(47.5)$ & $59(38.3)$ & $101(43.5)$ & $28(23.2)$ \\
\hline $6-7$ & $22(7.5)$ & $13(5.6)$ & $27(26.7)$ & $64(41.6)$ & $90(38.8)$ & $52(51.2)$ \\
\hline$>7$ & $8(2.7)$ & $10(4.3)$ & $4(4.0)$ & $21(13.6)$ & $15(6.5)$ & $19(15.7)$ \\
\hline \multicolumn{7}{|c|}{ Rationale for conservative treatment, $\mathrm{n}(\%)$} \\
\hline Comorbidities and contraindications & $227(77.0)$ & $140(59.8)$ & $45(44.5)$ & $43(27.9)$ & $75(32.3)$ & $33(27.3)$ \\
\hline
\end{tabular}

ERM, epiretinal membrane; NIEC, national investigation of ERM care.

and protocols for conservative treatments. Respondents most often cited "comorbidities and contraindications" $(49.5 \% ; 563 / 1,137)$ as the major reason for choosing conservative treatments. SES was more commonly cited as the main reason for choosing conservative treatment in Western regions, especially the South-Western regions $(67.8 \% ; 82$ of 121$)$ than it was in Eastern regions $[10.5 \%$ (31 of 295); OR: 17.91; 95\% CI: 10.51-30.50; $\mathrm{P}<0.001$ ]. Respondents in the Western regions seldom cited "choice of vitrectomy with ERM removal and ILM peeling", especially the South-Western region $(20.1 \% ; 31$ of 154$)$ than the Eastern region (77.3\%; 228 of 298) (Table 2).

\section{Discussion}

According to the data collected from the national ophthalmologist-oriented questionnaire, the proportion of monthly admissions and preoperative and postoperative care varied greatly depending on geographic locations in China.

Previous studies have shown that different factors are associated with the prevalence of ERM, including age, gender, and ethnicity. Ogihara et al. reported that a prognosis of ERM was closely associated with older age, female gender, myopia, and hyperlipidemia (11). Zhu et al. found that the incidence of ERM was associated with 


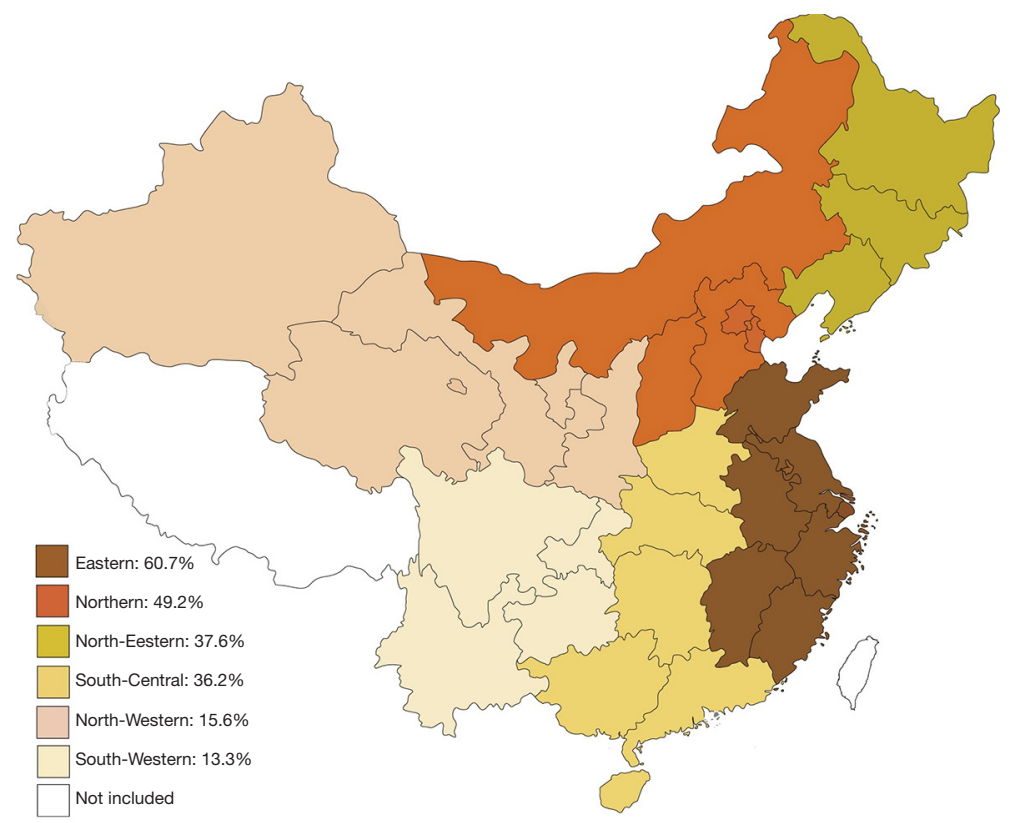

Figure 1 Comparison of the monthly admissions of ERM patients in different regions according to the NIEC questionnaire. ERM, epiretinal membrane; NIEC, national investigation of ERM care.
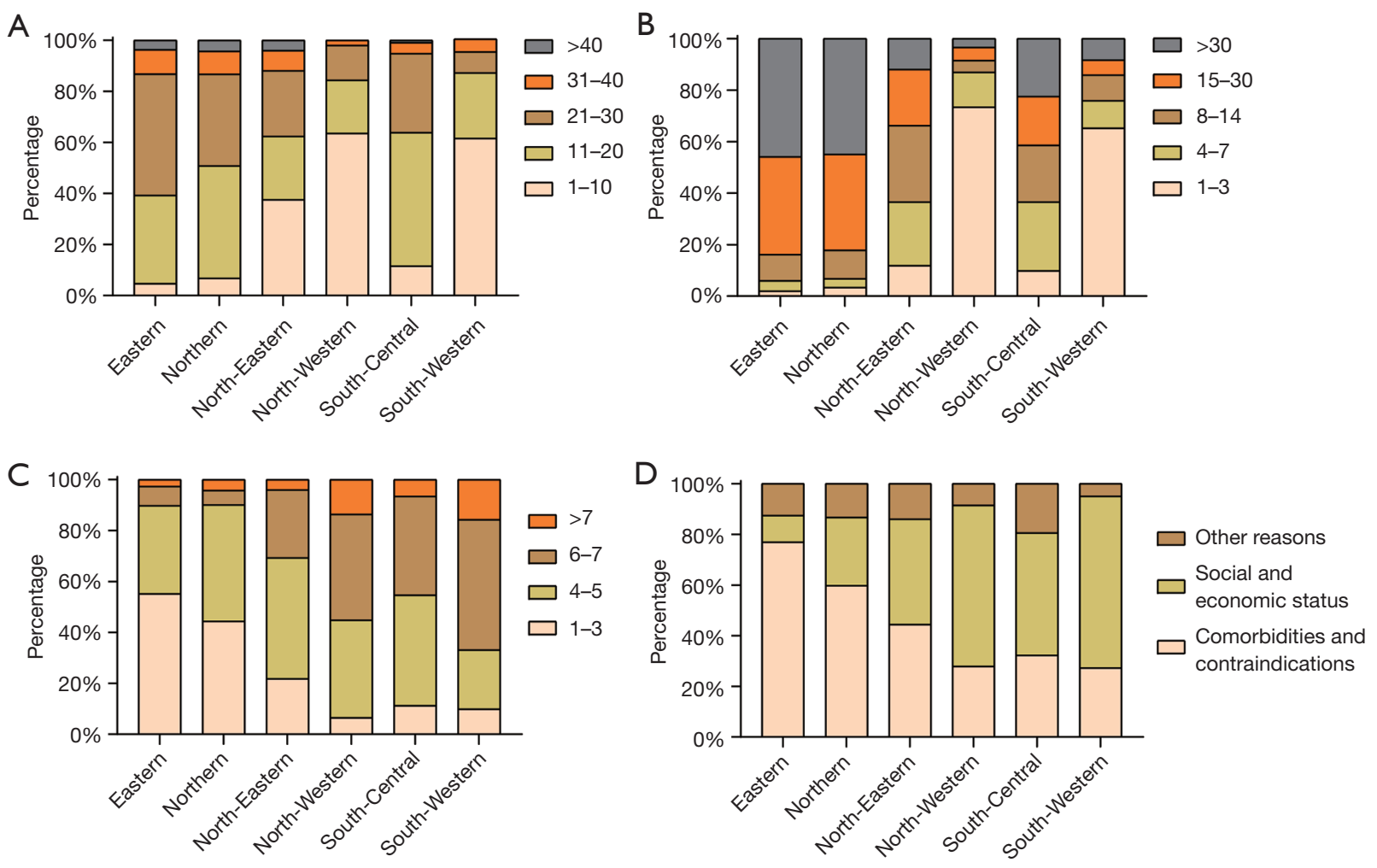

Figure 2 Characteristic variations of ERMs in different regions. (A) The different proportions of monthly admissions; (B) the distribution of percentages of preoperative waiting time; (C) the distribution of the length of hospitalization; (D) the distribution of the choice of conservative treatment. ERMs, epiretinal membranes. 
diabetes and a higher level of education (7). Conversely, another study conducted by $\mathrm{Bu}$ et al. identified age and hypertriglyceridemia as the risk factors of developing ERM (12). The different prevalence of ERM in these studies could be attributed to different population compositions and social status. In the present study, a national investigation of ERM care was conducted to improve the epidemiological dataset and medical treatment.

Our study examined the monthly admissions for ERM rather than its incidence, which represents a slightly different approach to previous studies. Patients suffering from ERM might not attend hospitals for specific reasons. Thus, our findings are directly related to the medical condition in China. The proportions of monthly admissions of over 20 patients varied greatly among different regions (see Table 2 and Figure 1). The South-Western and NorthWestern China regions accounted for a smaller proportion of admissions (13.3\% and 15.6\%), compared to the Eastern China region $(60.7 \%)$. The gross domestic product level of the Eastern China region is relatively higher than that of the Western region. Thus, our data showed that lower SES was associated with fewer admissions. The lower SES of the Western region might result in fewer hospitalizations and limited access to medical care. Additionally, some patients might choose a conservative treatment rather than surgery at hospital due to poverty.

The findings in this study also showed the differences between preoperative and postoperative care in each region. Patients in the Eastern and Northern regions waited longer for surgery than those in the Western regions. Indeed, a majority of patients in the Eastern region waited over 30 days for surgery. A large number of patients might have undergone surgery in the developed regions due to better medical care and the higher economic income of those regions. The top hospitals in the developed area were always full of patients and had a high number of admissions and insufficient operating rooms. Notably, ERM often occurs in elderly individuals, especially those with comorbidities and anticoagulant agents. Thus, early operations might improve prognosis and life quality. Expanding the hospital level of top hospitals and improving the medical care offered by the other hospitals could help patients flow from high-level hospitals to other hospitals for early operations.

The most common rationale in the lower SES regions for the conservative treatment of ERM was economic issues. Patients in remote districts, such as the Western region, chose a conservative treatment due to economic issues. However, increased comorbidities were the primary consideration in most areas, especially the Eastern region. Surgery for ERMs is important to improve patients' longterm visual condition (13). The peeling of ILM is applied as an accessory procedure to improve the curative effect, (14) and results in a lower recurrence rate (15). However, the choice of vitrectomy with ERM removal and ILM peeling was very unpopular in the Western regions. Doctors in the Western regions preferred a more conservative approach, which may have been informed by inadequate surgical guidelines (see Table 2). Optical coherence tomography angiography (OCTA)-based preoperative imaging, quantification of choroidal vasculature and perfusion state in patients with ERMs are important. Significant diurnal variations in patients with symptomatic ERM were reported and time of day should be considered when comparing longitudinal follow-up OCTA data (16).

This study has certain advantages. The web-based questionnaire was distributed by a specified website, email or mobile devices to particular ophthalmologists simultaneously to maximize response and completion rates and control responder bias. The respondents comprised ophthalmologists instead of the more commonly used patients to enable us to examine general medical data. The findings of this study will assist in the formulation of medical policies, and should provide insights into healthcare environments across China.

The study had some limitations. First, the data might not be suitable for the entire country. We used a strict multistage probability sampling procedure to reflect the current situation, and our respondents comprised over 1,000 surgeons; however, not every surgeon specifically treats ERMs. The medical data gathered from the doctors via the questionnaire may also be subjective, which further minimizes our responder bias. In terms of the subjective bias in our study, 1,065 doctors from 180 hospitals were included in this study. The answers were nearly the same by the participants of 1 hospital, indicating low subjectivity bias. Additionally, we could not collect information on patients' discharge destinations after they left the emergency department; thus, the entire hospitalization period may not have been estimated precisely. Finally, unlike in a similar questionnaire from Australia (17), our questionnaire did not include any specific scores to estimate socioeconomic status in China. Consequently, we had to use income data from the National Bureau of Statistics of China as a measure of SES. Lastly, the study of intraoperative dyeing usage for staining ILM was not included in this questionnaire due to the availability problem of dyes in mainland China $(18,19)$. 
In conclusion, the monthly admissions and preoperative and postoperative care of ERM patients varied significantly across different regions in China. Generally, the monthly patient admissions numbers were relatively lower in the Western China region compared to the Eastern region. However, patients in the Eastern China region have longer preoperative waiting times and shorter hospital stays.

\section{Acknowledgments}

We gratefully acknowledge the contributions of all the ophthalmologist respondents who participated in the current study.

Funding: None.

\section{Footnote}

Reporting Checklist: The authors have completed the SURGE reporting checklist. Available at http://dx.doi. org/10.21037/atm-21-2722

Data Sharing Statement: Available at http://dx.doi. org/10.21037/atm-21-2722

Conflicts of Interest: All authors have completed the ICMJE uniform disclosure form (available at http://dx.doi. org/10.21037/atm-21-2722). The authors have no conflicts of interest to declare.

Ethical Statement: The authors are accountable for all aspects of the work in ensuring that questions related to the accuracy or integrity of any part of the work are appropriately investigated and resolved. The study was conducted according to the Declaration of Helsinki (as revised in 2013) and was approved by the Institutional Ethics Board of Shanghai General Hospital (No. [2020]92) and individual consent for this cross-sectional survey was waived.

Open Access Statement: This is an Open Access article distributed in accordance with the Creative Commons Attribution-NonCommercial-NoDerivs 4.0 International License (CC BY-NC-ND 4.0), which permits the noncommercial replication and distribution of the article with the strict proviso that no changes or edits are made and the original work is properly cited (including links to both the formal publication through the relevant DOI and the license). See: https://creativecommons.org/licenses/by-nc-nd/4.0/.

\section{References}

1. Folk JC, Adelman RA, Flaxel CJ, et al. Idiopathic Epiretinal Membrane and Vitreomacular Traction Preferred Practice Pattern((R)) Guidelines. Ophthalmology 2016;123:P152-81.

2. Mao J, Xu Z, Lao J, et al. Assessment of macular microvasculature features before and after vitrectomy in the idiopathic macular epiretinal membrane using a grading system: An optical coherence tomography angiography study. Acta Ophthalmol 2021. [Epub ahead of print]. doi: 10.1111/aos.14753.

3. Coltrini D, Belleri M, Gambicorti E, et al. Gene expression analysis identifies two distinct molecular clusters of idiopatic epiretinal membranes. Biochim Biophys Acta Mol Basis Dis 2020;1866:165938.

4. Sun Y, Zhou R, Zhang B. With or without internal limiting membrane peeling for idiopathic epiretinal membrane: A Meta-Analysis of Randomized Controlled Trials. Retina 2020. [Epub ahead of print]. doi: 10.1097/ IAE.0000000000003076.

5. Sato T, Mori R, Takahashi S, et al. Anatomical and functional changes in paravascular abnormalities after epiretinal membrane removal. Graefes Arch Clin Exp Ophthalmol 2019;257:1823-9.

6. Bae JH, Song SJ, Lee MY. Five-Year Incidence and Risk Factors for Idiopathic Epiretinal Membranes. Retina 2019;39:753-60.

7. Zhu XF, Peng JJ, Zou HD, et al. Prevalence and risk factors of idiopathic epiretinal membranes in Beixinjing blocks, Shanghai, China. PLoS One 2012;7:e51445.

8. Huang Q, Li J. With or without internal limiting membrane peeling during idiopathic epiretinal membrane surgery: A meta-analysis. PLoS One 2021;16:e0245459.

9. Vogt D, Stefanov S, Guenther SR, et al. Comparison of Vitreomacular Interface Changes in Myopic Foveoschisis and Idiopathic Epiretinal Membrane Foveoschisis. Am J Ophthalmol 2020;217:152-61.

10. Tang X, Wang S, Zhan S, et al. The Prevalence of Symptomatic Knee Osteoarthritis in China: Results From the China Health and Retirement Longitudinal Study. Arthritis Rheumatol 2016;68:648-53.

11. Ogihara T, Mizoi K, Kamioka H, et al. Physiological Roles of ERM Proteins and Transcriptional Regulators in Supporting Membrane Expression of Efflux Transporters as Factors of Drug Resistance in Cancer. Cancers (Basel) 2020;12:3352.

12. Bu SC, Kuijer R, Li XR, et al. Idiopathic epiretinal 
membrane. Retina 2014;34:2317-35.

13. Iuliano L, Fogliato G, Gorgoni F, et al. Idiopathic epiretinal membrane surgery: safety, efficacy and patient related outcomes. Clin Ophthalmol 2019;13:1253-65.

14. Díaz-Valverde A, Wu L. To Peel or Not to Peel the Internal Limiting Membrane in Idiopathic Epiretinal Membranes. Retina 2018;38 Suppl 1:S5-S11.

15. Chang WC, Lin C, Lee CH, et al. Vitrectomy with or without internal limiting membrane peeling for idiopathic epiretinal membrane: A meta-analysis. PLoS One 2017;12:e0179105.

16. Rommel F, Siegfried F, Sochurek JAM, et al. Mapping diurnal variations in choroidal sublayer perfusion in patients with idiopathic epiretinal membrane: an optical coherence tomography angiography study. Int J Retina Vitreous 2019;5:12.

Cite this article as: $\mathrm{Xu} M$, Zhou M, Sun X, Shi X. Geographic variations in idiopathic epiretinal membranes in China. Ann Transl Med 2021;9(11):938. doi: 10.21037/atm-21-2722
17. Sajjad MA, Holloway-Kew KL, Mohebbi M, et al. Association between area-level socioeconomic status, accessibility and diabetes-related hospitalisations: a crosssectional analysis of data from Western Victoria, Australia. BMJ Open 2019;9:e026880.

18. Kwok AK, Lai TY, Li WW, et al. Trypan blue- and indocyanine green-assisted epiretinal membrane surgery: clinical and histopathological studies. Eye (Lond) 2004;18:882-8.

19. Tognetto D, De Giacinto C, D'Aloisio R, et al. The Combination of Trypan Blue and Brilliant Blue G-Assisted Vitrectomy for Macular Pucker: Histopathological Findings. Ophthalmologica 2018;239:167-75.

(English Language Editor: L. Huleatt) 